\title{
Mechanochemically Initiated Polymerizations III. Mechanochemical Polymerization of Acetonitrile by Vibratory Grinding
}

\author{
Cleopatra Vasiliu Oprea, Marcel PoPA, and Nicolae HurduC \\ Department of Organic and Macromolecular Chemistry, \\ Polytechnic Institute of Jassy, 23 August Street, \\ $N^{\circ} 11,6600$ Jassy, Rumania
}

(Received June 20, 1983)

\begin{abstract}
The paper shows evidence for the capacity of impact mechanical energy released by vibratory grinding to initiate polymerization reactions of acetonitrile. The influence of certain factors (reaction duration, gaseous atmosphere, medium polarity) was studied. The resultant products were characterised by spectral analyses; some specific properties determined by the presence of metallic atoms in the polymer chain are also evidence in support of this ability.

KEY WORDS Acetonitrile / Poly(acetonitrile) / Mechanochemistry /

Vibratory Grinding / Mechanochemical Polymerization /
\end{abstract}

Recent studies $^{1-3}$ have demonstrated the capacity of mechanical energy to activate vinyl monomers polymerization reactions without activation additives. This is evident in the synthesis of homo- and copolymers of acrylonitrile, styrene and vinyl acetate. The properties of the resultant polymers were found sometimes to differ from those of their homologues obtained by conventional methods.

The presence of conjugated $-\mathrm{C}=\mathrm{N}$ - double bonds ${ }^{3}$ in the main chain of mechanochemically synthesised poly(acrylonitrile) led to the conclusion that the $-\mathrm{C} \equiv \mathrm{N}$ triple bond participates in the polymerization reaction.

Several literature references show the possibility of polymerization of nitriles in the absence of initiators, at high temperatures and pressure $(7-20 \mathrm{kbar}),{ }^{4}$ to yield micromolecular triazinic products mixed with insoluble and infusible polymers resulting at high reaction rates. Solid state polymerization of nitriles was also possible by $\gamma$ - and X-rays irradiation. ${ }^{5,6}$

Interesting results were obtained by the solid state polymerization of nitriles under high pressure and intense shear stress. ${ }^{7}$ Under these conditions, homolytic breakage of some chemical bonds took place and free radicals, initiating the polymerization of $-\mathrm{C} \equiv \mathrm{N}$ group, resulted. ${ }^{8,9}$

The synthesis of the polymers of some aromatic and aliphatic nitriles by ionic mechanism should also be mentioned. By using the complexes of metals belonging to II-IV and VIII groups for polymerization initiation, reaction products consisting of two fractions were obtained: an insoluble and infusible polymer in a significant amount and a soluble macromolecular compound in a lesser amount. ${ }^{10-13}$

The present paper deals with the polymerization of the $-\mathrm{C} \equiv \mathrm{N}$ triple bond by the action of mechanical energy, under vibratory grinding conditions. The influence of certain factors on this type of polymerization were studied and information on the reaction mechanism was obtained.

\section{EXPERIMENTAL}

The monomer was purified by distillation and drying on molecular sieves. The syntheses were performed on a vibratory mill (Labowi 
LS-60 type) operating at a frequency of $25 \mathrm{~Hz}$ and vibration amplitude of $2-4 \mathrm{~mm}$. The mill vessel (41) was manufactured of $\mathrm{V}_{2} \mathrm{~A}$ steel $(73.75 \% \mathrm{Fe}, 18 \% \mathrm{Cr}, 8 \% \mathrm{Ni}, 0.1 \% \mathrm{Nb}, 0.1 \%$ $\mathrm{Ti}, 0.02 \% \mathrm{C}$ ) and provided with valves for maintaining the required gas atmosphere and a thermostatting jacket. The working temperature was kept at $18 \pm 2^{\circ} \mathrm{C}$. The grinding balls (spherical, $\phi=9 \mathrm{~mm}$ ) were also made of $\mathrm{V}_{2} \mathrm{~A}$ steel and together weighed about $6 \mathrm{~kg}$.

The air was removed from the vessel containing the grinding balls by successive connection ( $3-4$ times) to a vacuum line and repeated flushing with nitrogen. The required amount of monomer $(30 \mathrm{~g})$ was then introduced to a filling ratio of $0.5 \%{ }^{2}$ The vessel was kept under nitrogen, except the experiments for elucidating the medium influence.

To study the influence of the medium polarity, $30 \mathrm{~g}$ of liquids with different dielectric constants $(\varepsilon)$ were introduced.

The obtained reaction product was extracted with methanol in a Soxhlet apparatus into the vessel to remove the unreacted monomer and the soluble fraction. The recovery of the soluble fraction from the extract was accomplished by vacuum distillation of the solvent. The two fractions (the soluble and the insoluble one) thus purified were dried under vacuum at $40^{\circ} \mathrm{C}$.

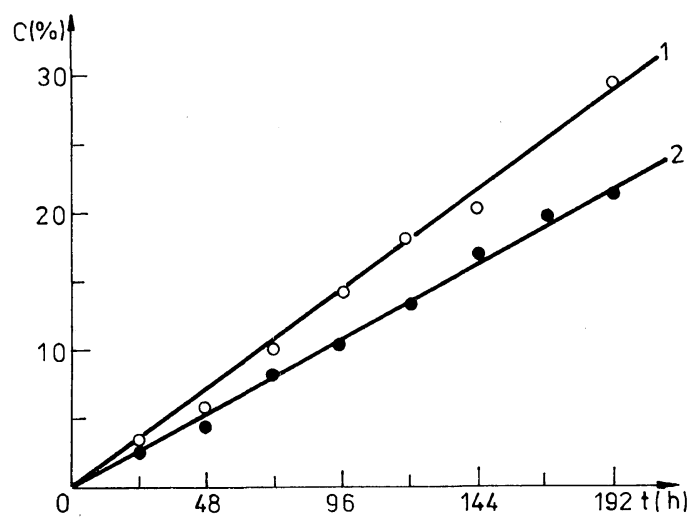

Figure 1. Variation in conversion with reaction time: 1 , in nitrogen; 2 , in air.
The reaction products were analysed by emission spectroscopy using a spectrometer Q24, Carl Zeiss Jena type, and by Mössbauer spectroscopy (Austin Texas Instrument). The Mössbauer apparatus scale was calibrated with sodium nitroferricyanide and the data were processed by an IBM 370/135 computer. Each spectrum was recorded for $24 \mathrm{~h}$.

IR spectra were recorded on a Perkin-Elmer spectrometer, using $\mathrm{KBr}$ pellets.

The nitrogen content and the calcination residue were determined by Kjeldhal's method and calcination at $1000^{\circ} \mathrm{C}$, respectively.

\section{RESULTS AND DISCUSSION}

Early experiments ${ }^{1-3}$ indicated that to obtain high polymer conversions, the synthesis duration of at least $24 \mathrm{~h}$ was necessary. A period of $192 \mathrm{~h}$ was the upper limit for this study. The influence of this period on reaction efficiency was expressed in terms of the variation in conversion.

The results presented in Figure 1 show a linear increase in conversion with time, regardless of the nature of the gaseous atmosphere in the reaction vessel. It should be noticed that

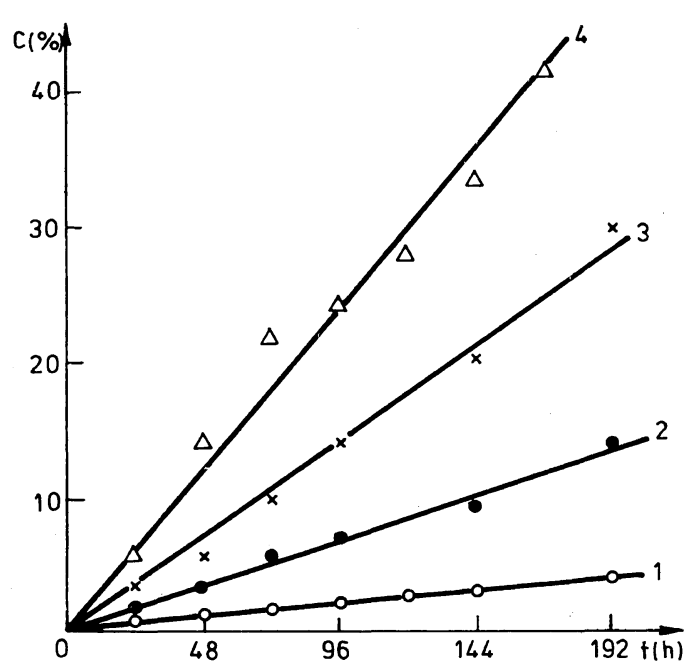

Figure 2. Variation in conversion with reaction time in media of different polarities: 1 , hexane; 2 , methanol; 3 , nitrogen; 4 , water. 
Table I. Influence of medium polarity on the efficiency of acetonitrile polymerization $(t=144 \mathrm{~h})$

\begin{tabular}{lccc}
\hline & \multicolumn{3}{c}{ Dielectric constant } \\
Reaction system & \multicolumn{2}{c}{$(\varepsilon)$} & Conversion \\
\cline { 2 - 3 } & $\begin{array}{c}\text { Liquid } \\
\text { medium }\end{array}$ & $\begin{array}{c}\text { Reaction } \\
\text { mixture }\end{array}$ & $\%$ \\
\hline $\begin{array}{c}\text { Acetonitrile +hexane } \\
(1: 1 \text { weight ratio) }\end{array}$ & 2.01 & 26.38 & 3.2 \\
$\begin{array}{c}\text { Acetonitrile }+ \text { methanol } \\
(1: 1 \text { weight ratio) }\end{array}$ & 33.6 & 35.52 & 9.5 \\
$\begin{array}{c}\text { Acetonitrile }\left(\mathrm{N}_{2}\right) \\
\text { Acetonitrile }+ \text { water } \\
(1: 1 \text { weight ratio })\end{array}$ & 38.0 & 38.0 & 20.2 \\
\hline
\end{tabular}

for syntheses carried out in the presence of air oxygen, the conversion was always smaller than that obtained in the nitrogen atmosphere.

Significant conclusions were obtained from the data obtained using liquids of different polarities as polymerization media (liquidmonomer, 1:1 weight ratio). Figure 2 indicates the linear increase in conversion with time, the reaction efficiency to increase simultaneously with $\varepsilon$.

Syntheses, carried out in the absence of liquids of different polarities, have conversion values within the range of those obtained with methanol (Figure 2, curve 2) and water (Figure 2 , curve 4). This is supported by the value of the dielectric constant of acetonitrile $(\varepsilon=38)$ which is higher than those of methanol $(\varepsilon=33.6)$ but smaller than those of water $(\varepsilon=80.3)$.

The effects of the average dielectric constant of the whole reaction mixture on conversion is summarized in Table I.

The results led to the conclusion that the influence of the liquids with increasing dielectric constant on the process efficiency may be explained only by the presence of certain ionic active centers in the mechanism of acetonitrile polymerization reaction.

An ionic mechanism of polymerization may be accepted considering that in the $\mathrm{V}_{2} \mathrm{~A}$ steel, some transitional metals such as $\mathrm{Fe}, \mathrm{Ni}, \mathrm{Cr}, \mathrm{Ti}$
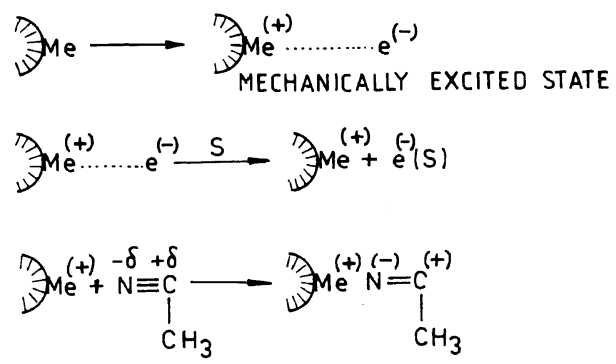

Figure 3. Initiation of the mechanochemical polymerization of acetonitrile.

are present. Under the action of repeated intense schocks and corrossive action of the liquid, the kinetic energy developed in the system is consumed, at least partially, exciting the electrons on the surface layers of the metal atoms, thus promoting electron emission. Mechanoexcited states consisting about in this way become active centers, as shown in the schema in Figure 3. The metallic ions become capable of initiating the polymerization of the $-\mathrm{C} \equiv \mathrm{N}$ triple bond.

Under these conditions, the propagation reaction may take place either at the ionic pair, or at the cationic end. The first mechanism is less probable, since it is well known that an anionic center is inactivated by proton donating substances and hydrogen transfer. If an anionic reaction took place the conversions obtained in the presence of water would have been inferior to those obtained from acetonitrile ground in the absence of this liquid. The experimental results show contrary effects and support a cationic mechanism. This also explains why the efficiency of the reaction increases with the dielectric constant of the medium and its electron solvation capacity.

Even the electron may be solvated by acetonitrile to a higher extent than by methanol $\left(\varepsilon_{\mathrm{CH}_{3} \mathrm{OH}}<\varepsilon_{\mathrm{CH}_{3} \mathrm{CN}}\right)$. Consequently, the continuously higher conversions obtained without methanol appear to be normal.

The mechanism occurring by the free cation is also proved by the presence of chemically bonded metal in the structure of the reaction 


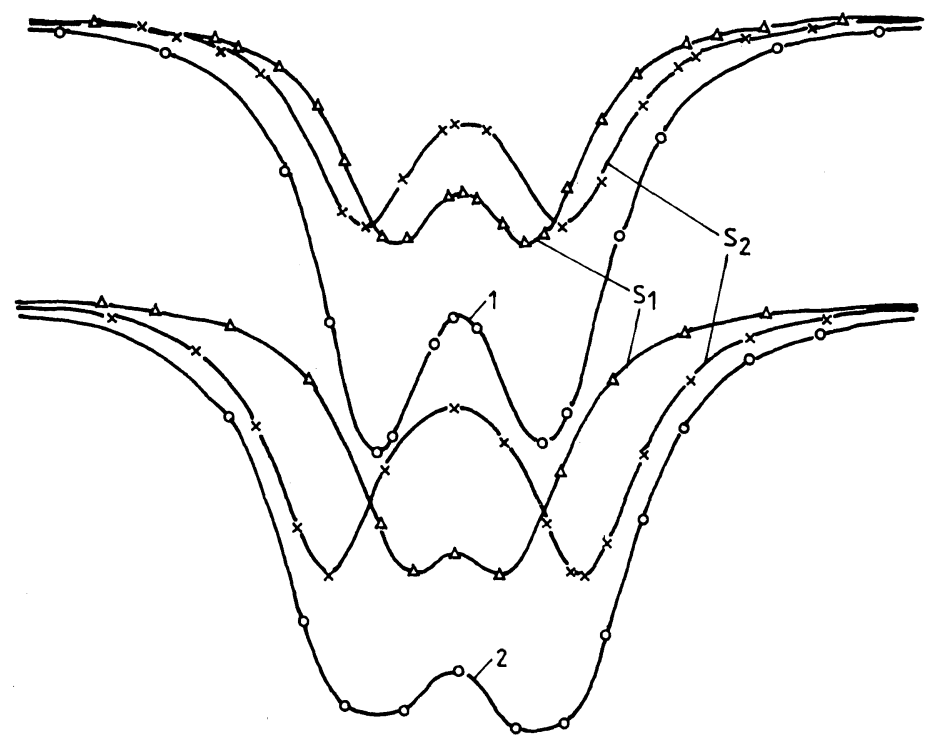

Figure 4. Mössbauer spectra of some polymerization products of acetonitrile, in nitrogen (72h): 1 , soluble fraction; 2 , insoluble fraction.

products, as demonstrated by the results of spectral analyses. Thus, emission spectroscopy showed that mechanochemically synthesised poly(acetonitrile) contains within its structure $\mathrm{Ni}, \mathrm{Cr}, \mathrm{Ti}$ at a $10^{-2}-10^{-3} \mathrm{wt} \%$ concentration, but especially $\mathrm{Fe}$ in amounts greater than $1 \mathrm{wt} \%$. The percent of chemically bonded Fe was accurately determined by Mössbauer spectroscopy and the spectra are given in Figure 4. The presence of iron in the reaction products, both in the insoluble polymer and in the soluble fraction (the later with a $1.8 \mathrm{wt} \%$ metal content) was proved by the parameters calculated from the Mössbauer spectra given in Table II.

The ionic mechanism of the reaction and the conclusion that the reaction products are mixtures of soluble and insoluble fractions are in agreement with literature data. ${ }^{10-13}$

By vibratory grinding of acetonitrile in the presence of liquids of different polarities (hexane, methanol) a fraction soluble in methanol (mp under $100^{\circ} \mathrm{C}$ ) and one insoluble and infusible were obtained.

Variation in the amount of the soluble fraction with grinding duration is shown in Figure
Table II. Parameters calculated from Mössbauer spectra

\begin{tabular}{|c|c|c|c|c|}
\hline \multirow[t]{2}{*}{ Sample ${ }^{a}$} & $\begin{array}{l}\text { Isometric } \\
\text { shift } \\
(\delta)\end{array}$ & $\begin{array}{l}\text { Quadrupolar } \\
\text { splitting } \\
\left(\Delta E_{\mathrm{q}}\right)\end{array}$ & \multirow[t]{2}{*}{$\begin{array}{c}\text { Mössbauer } \\
\text { effect } \\
(\varepsilon)\end{array}$} & \multirow{2}{*}{$\frac{\mathrm{Fe}}{\%}$} \\
\hline & $\mathrm{mm} \mathrm{s}^{-1}$ & $\mathrm{~mm} \mathrm{~s}^{-1}$ & & \\
\hline \multirow{2}{*}{$\begin{array}{l}\text { Soluble } \\
\text { fraction }\end{array}$} & 0.0517 & 0.3030 & 0.9 & \multirow{2}{*}{1.8} \\
\hline & 0.0576 & 0.4417 & 0.9 & \\
\hline \multirow{2}{*}{$\begin{array}{l}\text { Insoluble } \\
\text { fraction }\end{array}$} & -0.0946 & 0.5453 & 0.8 & \multirow{2}{*}{1.45} \\
\hline & -0.0873 & 0.2283 & 0.65 & \\
\hline
\end{tabular}

a $t=72 \mathrm{~h}$; inert medium $\left(\mathrm{N}_{2}\right) ; \eta=0.5 \% ; T=18 \pm 2^{\circ} \mathrm{C}$.

5. The accumulation of greater amounts of soluble products was evident in the synthesis with methanol and the conversion reached $2.5 \%$ after $144 \mathrm{~h}$ (curve 2 ).

As confirmed by IR spectroscopy, the soluble fraction is a poly(acetonitrile). Thus, in the IR spectra (Figure 6), a series of characteristic bands were found at $2900 \mathrm{~cm}^{-1}\left(-\mathrm{CH}_{3}\right.$ group), 1450 and $1370 \mathrm{~cm}^{-1}$ (-C-H group), and the most important band, appearing at $1600-1700 \mathrm{~cm}^{-1}$, was attributed to the $-\mathrm{C}=\mathrm{N}-$ bond in the conjugated systems. The 
disappearance of the $-\mathrm{C} \equiv \mathrm{N}$ band at $2240 \mathrm{~cm}^{-1}$ may be attributed, in the present case, to the conversion of these groups during polymerization.

The band at $1600-1700 \mathrm{~cm}^{-1}$ also characterises the IR spectrum of the insoluble fraction, but is weaker in intensity (Figure 6, curve 2).

IR spectroscopy data confirm the conclusion that, under the action of the mechanical energy, aliphatic nitriles may polymerize through the $-\mathrm{C} \equiv \mathrm{N}$ group.

The major component of the reaction product is an insoluble, infusible polymer, of dark brown color; the soluble fraction was lighter in color.

Attempts to solubilize the insoluble fraction in different solvents including those of high polarity (DMF, DMSO) were not successful. This fraction also showed high chemical stability toward strong hydroxides and mineral acids. The only exception was the case of $\mathrm{H}_{2} \mathrm{SO}_{4}\left(1.83 \mathrm{~g} \mathrm{~cm}^{-3}\right.$ density); on periodic heating the polymer was completely degraded.

To obtain soluble polymers of acetonitrile in common solvents, the synthesis of an acetonitrile-styrene copolymer was carried out (weight ratio in initial mixture, 2:1), in a nitrogen atmosphere for $96 \mathrm{~h}$. The reaction product was soluble in cold methanol (up to $60 \%$ ) and completely so in DMF. The copoly-

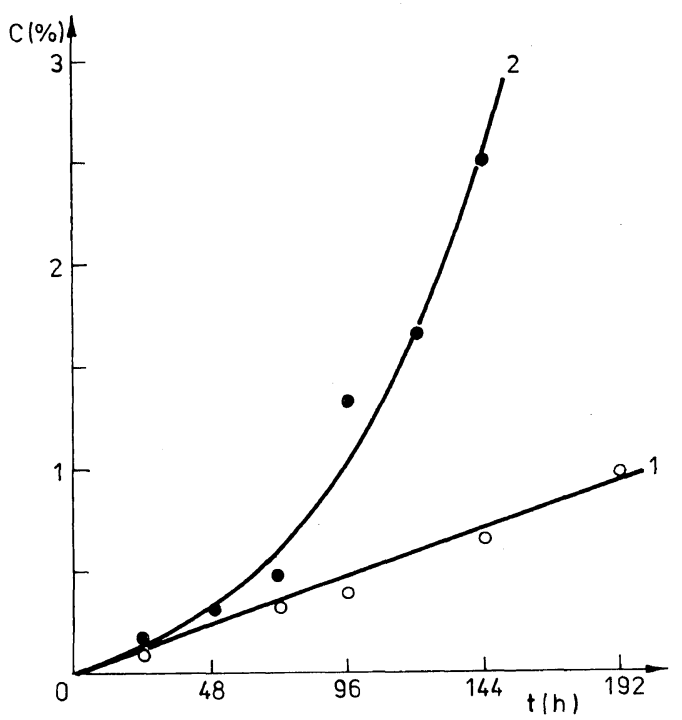

Figure 5. Variation in amount of the fraction soluble in methanol with reaction time, in different media: 1 , hexane; 2 , methanol.

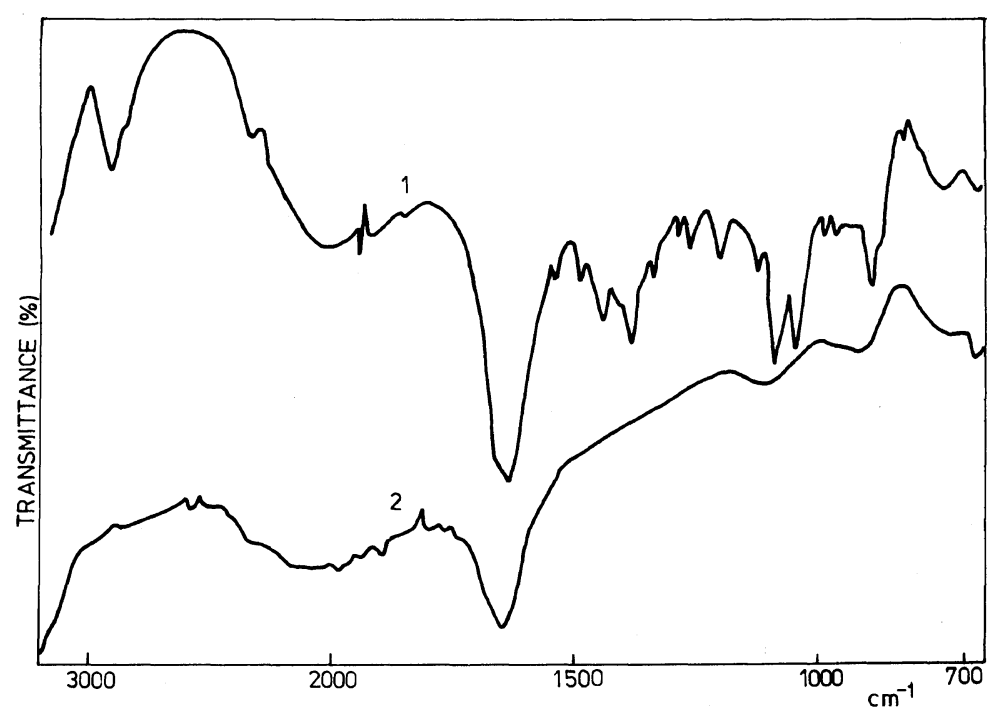

Figure 6. IR spectra of poly(acetonitrile) synthesized in the presence of hexane ( $72 \mathrm{~h})$ : 1 , soluble fraction; 2 , insoluble fraction. 


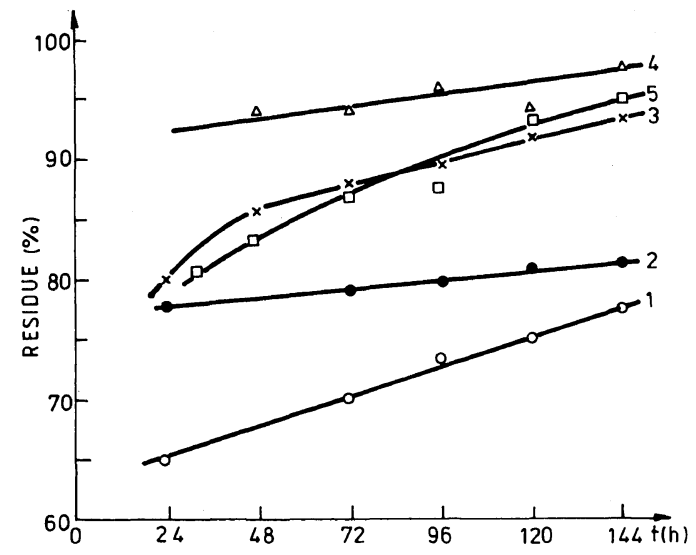

Figure 7. Variation in the thermostable product content (calcination residue at $1000^{\circ} \mathrm{C}$ ) with synthesis duration: 1 , in hexane; 2 , in methanol; 3 , in nitrogen; 4 , in water; 5 , in air.

merization conversion was $40 \%$.

One notable property of the insoluble fraction of poly(acetonitrile) was its high thermal stability. Thus, all synthesized polymers yielded at least $60 \%$ thermostable products by calcination at $1000^{\circ} \mathrm{C}$. Variation in the thermostable products with synthesis duration is shown in Figure 7. The thermostable product increased with the dielectric constant of the polymerization medium. The most thermostable polymer was that obtained in the presence of water.

Our data imply the capacity of mechanical energy to initiate the polymerization of nitriles. Based on the experimental results, the reaction seems to proceed more probably by a free cationic ion mechanism. The reaction is disturbed by oxygen, not directly, but by the simultaneous stimulation of mechanodegradation occurring with mechanochemical synthesis. This secondary reaction proceeds by a radical mechanism and oxygen acts as an acceptor to prevent the recombination of macroradicals. Consequently, the content of the low molecular weight products increases (the soluble fraction). These products are solubilized by the extraction agent used for the polymer purification. The mechanochemical degradation accompanying the polymerization reaction was found in all the experiments. ${ }^{1-3}$

As shown by IR spectroscopy, the soluble fraction of the obtained products is a poly(acetonitrile):

$$
\begin{aligned}
& (\underset{\mathrm{CH}}{\mathrm{C}}=\mathrm{N})_{n} \\
&
\end{aligned}
$$

Compared to the chemically synthesized poly(acetonitrile), ${ }^{10-13}$ the products obtained by mechanochemical synthesis (especially the insoluble fraction) show some special properties due to the presence of chemically bonded metal atoms in their structures. Their insolubility and infusibility, particularly their outstanding thermostability, are characteristic of macromolecular complexes. ${ }^{14}$ It may be concluded that the insoluble fraction is possibly included in this class of polymers due to the presence of some metals, especially iron, in significant amounts and chemically bonded.

\section{CONCLUSIONS}

1. The polymerization of aliphatic nitriles was carried out under the influence of mechanical energy generated by vibratory milling.

2. The main factors governing the mechanochemical polymerization reaction are the nature of the gaseous atmosphere and polarity of the reaction medium.

3. The obtained products incorporate into their structures metallic atoms from the walls of the apparatus.

4. The acetonitrile-based polymers are almost completely insoluble, infusible and thermostable.

5. Acetonitrile-based copolymers (with styrene) may be synthesized by vibratory milling; the copolymers take on increased solubility, compared to poly(acetonitrile).

\section{REFERENCES}

1. C. I. Simionescu, C. Vasiliu-Oprea, and C. Negulianu, Eur. Polym. J., 15, 1037 (1979). 
2. C. Vasiliu-Oprea and M. Popa, Angew. Makromol. Chem., 90, 13 (1980).

3. C. Vasiliu-Oprea and M. Popa, Angew. Makromol. Chem., 92, 73 (1980).

4. I. B. Johns, Polym. Prepr. Am. Chem. Soc., Div. Polym. Chem., 5, 239 (1964).

5. S. Okamura, K. Hayashi, M. Yamamoto, and Y. Nakamura, Kogyo Kagaku Zasshi, 65, 728 (1962).

6. S. H. Chen, N. Colthup, W. G. Deichert, and R. L. Welb, J. Polym. Sci., 45, 247 (1960).

7. I. E. Pahomova, Izd. AN. SSSR, Him., 857 (1972).

8. I. E. Pahomova, Vysokomol. Soedin. Ser. A, 12, 1849 (1970).
9. A. A. Petrov, M. G. Gonikberg, and D. N. Aneli, Vysokomol. Soedin. Ser. A, 10, 875 (1968).

10. D. Wöhrle and G. Helling, J. Polym. Sci., Polym. Symp., 42, 443 (1973).

11. V. A. Kabanov, V. P. Zubov, V. P. Kovalova, and V. A. Kargin, J. Polym. Sci., C, No. 4, 1009 (1963).

12. V. A. Kargin, V. A. Kabanov, V. A. Zubov, and A. B. Zezin, Dokl. Akad. Nauk SSSR, 139, 605 (1961).

13. V. A. Zubov and V. A. Kargin, Vysokomol. Soedin., 8, 795 (1966).

14. E. M. Natanson and Z. R. Ul'berg, "Kolloidnie Metally i Metallopolimeri," Naukova Dumka, Kiev, 1971, p 34. 